\title{
Study on Policy Framework for Distributed Energy of China
}

\author{
Guo Lei \\ State Grid Energy Research Institute (Beijing China)
}

\begin{abstract}
Distributed energy (DE) has characteristic of energy efficient using and saving and has become the one important way of China's energy development. In recent years, DE has been rapid developed, but also faces some constraints in China. This paper analyzes the role of DE in China's energy supply system, studies the constraints of DE, and put forward the policy framework for DE in China.
\end{abstract}

Keywords: Distributed Energy, Energy Supply, Policy Framework

\section{Introduction}

While the pressure on climate change and environmental protection is increasing in China, the transformation in energy development mode, the adjustment of the energy structure, and the improvement of energy efficiency have become the focus of energy development. China has not yet developed specialized policies for $\mathrm{DE}$, which has affected the development of DE.

This paper analyzes the constraints of DE development and study on the policy framework for DE to support the establishment and improvement of distributed energy-related policy in China.

\section{DE's role in China's energy supply system}

China has the characteristics of the dual structure of urban and rural development, which makes China's urban and rural energy demand, supply modes and features be great differences.

According to the characteristics of China's urban and rural areas, the development of DE needs to adapt to local conditions.

\subsection{Developing strategy of DE in ur- ban areas}

Most of China's industrial and economic output is concentrated in the city, which the prefecture-level city's industrial output value reached 24.7 trillion Yuan and $63 \%$ of total GDP at the end of $2008^{[1]}$. Energy is also consumed in urban areas.

The residential energy consumption will significantly increase, with China's industrial structure adjustment and upgrade of consumption structure in the future.

In order to improve the energy utilization efficiency, reduce urban energy consumption and achieve targets of energy conservation, the clean and environmentally friendly distributed energy should be developed in urban areas. Especially, CCHP will have the broad market, since the residence and commercial buildings, hospitals, public buildings, factories and stadiums need both power and heating or cooling in most urban areas of China. 
CCHP powered by natural gas can be properly developed in big or medium sized cities which have good gas grid and stable gas supply. The waste, landfill and biomass distributed energy, small PV and wind plants are also the focus of development in the same areas.

\subsection{Developing strategy of DE in ru- ral and remote areas}

DE could be developed in remote areas to solve the power supply. The centralized power supply in China's rural areas will require huge investments, which will result in higher electricity price. Small wind plant, solar photovoltaic and biomass are the most effective measure to solve the lack of electricity in remote areas in China. Especially for the western remote areas, DE can support the economic development in these areas by using the renewable energy resources.

\section{Status and constraints of DE in China}

\subsection{Status}

In recent years, the government of China has introduced a series of laws and policies to promote the development and utilization of renewable energy, which has also simulated the development of DE.

At the end of June 2012, the total installed capacity of DE accessed to $35 \mathrm{KV}$ and below grid was $34360 \mathrm{MW}$, including distributed hydropower accounted for $69.2 \%$ of total installed capacity, waste heat and residual gas comprehensive utilization accounted for $21.2 \%$, biomass accounted for $1.3 \%$, waste power plant accounted for $2.8 \%$; distributed photovoltaic accounted for $3.0 \%$, small wind power accounted for $1.4 \%$, and natural gas DE accounted for $1.1 \%$.

\subsection{Constraints}

Building PV and natural gas DE are still in the beginning stage, and the constraints of the development of DE include:

(1) Less the competition ability in the market.

The economy of DE is difficult to be reflected in the market, since the value of energy and environment can't be revealed in the pricing system in China. The unit capacity investment of $\mathrm{DE}$ is as 1.5-2 times as the conventional gas plants, which causes the price of DE much higher than coal-fired plants. The other reason is incentive polices for DE is not introduced in most areas of China.

(2) Shortage of the Natural gas supply for DE.

China's natural gas resources are insufficient resources, and the natural gas resources of China is only $1.5 \%$ of world resources. The consumption of natural gas was 87.45 billion cubic meters in $2009^{[2]}$, accounting for $4 \%$ of the total energy consumption, below the world average level. The shortage of natural gas supply limits the development of DE.

(3) Lack of access standard for DE.

China has not yet developed the unified access standard for DE to grid or low voltage distribution network, making DE access the grid difficultly.

(4) Lack of the reasonable price system and mechanisms.

Current power price system, which is suitable for centralized power system, can't be reflected the reasonable cost allocation and not includes the pricing principles of DE, which makes DE not be fully utilized and the development of DE be restricted. 


\section{China's policy framework for DE}

\subsection{Definition of distributed energy in China}

Firstly, Distributed energy systems should be based on the energy cascade utilization and renewable energy-based, which reflects the energy saving principles. Secondly, the total installed capacity of DE should be limited to a certain scale, emphasizing the local energy supply. Thirdly, DE should be connected to distribution grid.

\subsection{Development plan of DE}

Distributed renewable energy can be taken into national renewable energy planning. Natural gas DE needs to be included the country's new energy development plan, which adheres to the principles of energy conservation. Provincial plan of DE should be under the national planning guidance, which clears the development potential, objectives, key technologies and supporting measures in the province.

\subsection{Management and coordination policies}

The management coordination policy in the aspect of approval and pricing for DE between the energy authorities and other departments can be optimized further. The DE project, which applies for access to grid or preferential policies, can be approved by single government department.

\subsection{Unified national standard of grid- integration for DE}

In order to improve the reliability and economy of distributed energy, the national standard for DE accessing to the grid, including the technical standards and grid equipment configuration standards, should be set by the relevant government departments.

\subsection{Incentive policies}

The policies for DE should be introduced to guide and encourage the development of DE, which includes the subsidies policies of investment and operation of DE. The transactions between DE and other power consumer can be developed gradually inside the policies and laws, and DE companies will pay the electricity price and capacity price to the grid companies.

\section{Conclusion}

DE with the characteristics of high energy utilization efficiency, less pollution and lower energy consumption is the one of the important ways to improve the energy clean and efficient utilization. China needs to introduce the policy system for DE to guide and encourage the development of DE to achieve the goals of energy conservation.

\section{References}

[1] The national bureau of statistics, "China Statistical Yearbook," China statistics press, pp. 54-55, 2010.

[2] National Energy Administration, "China energy statistical yearbook," China statistics press, pp. 67-68, 2009. 